\title{
Cosmic Antiproton Sensitivity for the GAPS Experiment
}

\section{Field Rogers ${ }^{a, *}$ on behalf of the GAPS Collaboration}

(a complete list of authors can be found at the end of the proceedings)

\author{
${ }^{a}$ Massachusetts Institute of Technology, \\ 77 Massachusetts Avenue, Cambridge, MA 02139 USA \\ E-mail: frrogers@it.edu
}

The General Antiparticle Spectrometer (GAPS) experiment is a balloon payload designed to measure low-energy cosmic antinuclei during at least three $~ 35$-day Antarctic flights, with the first flight planned for December, 2022. With its large geometric acceptance and novel exotic atombased particle identification method, GAPS will detect $\sim 1000$ antiprotons per flight, producing a precision cosmic antiproton spectrum in the kinetic energy range of $0.03-0.23 \mathrm{GeV} / n$ at float altitude (corresponding to $0.085-0.30 \mathrm{GeV} / n$ at the top of the atmosphere). With these high statistics in a measurement extending to lower energy than any previous experiment, and with orthogonal sources of systematic uncertainty compared to measurements made using traditional magnetic spectrometer techniques, the GAPS antiproton measurement will be sensitive to physics including dark matter annihilation, primordial black hole evaporation, and cosmic ray propagation. The antiproton measurement will also validate the GAPS exotic atom technique for the antideuteron and antihelium rare-event searches and provide insight into models of cosmic particle attenuation and production in the atmosphere. This contribution demonstrates the GAPS sensitivity to antiprotons using a full instrument simulation, event reconstruction, and solar and atmospheric effects.

$37^{\text {th }}$ International Cosmic Ray Conference (ICRC 2021)

July 12th - 23rd, 2021

Online - Berlin, Germany

\footnotetext{
${ }^{*}$ Presenter
} 


\section{Introduction}

Cosmic antinucleus fluxes present an excellent channel for detection of new phenomena due to their relatively low astrophysical abundances [1]. Antiprotons constitute $<0.01 \%$ of cosmic particles at the top of Earth's atmosphere (TOA), and antideuterons and antihelium-3, whose fluxes are several orders of magnitude lower than antiprotons, are the subject of rare event searches. Lowenergy antiprotons in particular are sensitive to light dark matter (DM) annihilation, primordial black hole (PBH) evaporation, and Galactic and solar propagation models.

Various experiments and experimental techniques have been used to measured the antiproton spectrum at TOA. Recent measurements by AMS-02 [2], BESS [3, 4], and PAMELA [5] have provided information on the antiproton flux from kinetic energy (KE) of $\sim 0.2 \mathrm{GeV} / n$ to $450 \mathrm{GeV} / n$. Discussion is ongoing in the community related to a possible excess of $\sim 10-20 \mathrm{GeV} / n$ antiprotons measured by AMS-02, which has been interpreted as a signal of annihilating DM with mass $M_{D M}$ in the range of $40-130 \mathrm{GeV}$ [6-10], though the significance of this detection depends on the treatment of systematic errors and their correlation, which was not reported by AMS-02, e.g. [9, 11]. .

The General Antiparticle Spectrometer (GAPS) [12-15], a NASA Antarctic long-duration balloon (LDB) payload, will extend the precision cosmic antiproton spectrum to lower energies using a detection method with orthogonal sources of systematic uncertainty compared to traditional magnetic spectrometer techniques. The GAPS antiproton measurement will contribute new data to discussions of the possible AMS-02 antiproton excess. With high statistics at low energies, GAPS could constrain the falling low-energy edge of a primary DM spectral component.

Hawking radiation from evaporation of PBHs that may have formed in the early Universe would be a source of cosmic antiprotons [28]. The lifetime for PBHs formed with mass $M_{\star} \sim 5 \times 10^{14} \mathrm{~g}$ is $\sim 14 \mathrm{Gyr}$; any such PBHs would be evaporating in the present day. A local evaporating PBH population would contribute an antiproton flux broadly peaked at $\sim 0.5 \mathrm{GeV}$ at TOA [28], which could be observed in the GAPS antiproton measurement.

Uncertainties in models of cosmic ray propagation in the Galaxy represent one of the dominant systematic uncertainties associated with indirect DM searches. The astrophysical flux of cosmic antiprotons is dominated by secondary production in the Galaxy, and low-energy antiprotons are strongly deflected by magnetic fields. This makes low-energy antiprotons excellent probes of cosmic ray propagation models, as has been shown since the first calculation of secondary antiprotons [16].

The general applicability of the GAPS detection technique to new-physics searches using antiprotons was previously established [17]. This contribution uses a detailed detector simulation with full event reconstruction to assess the sensitivity of the GAPS instrument to cosmic antiprotons in its first flight and in the full GAPS exposure of 3 LDB flights.

\section{Antiparticle Identification with the GAPS Experiment}

The GAPS experiment is designed to detect low-energy ( $\mathrm{KE} \lesssim 0.25 \mathrm{GeV} / n)$ antinuclei using a novel exotic atom-based method for particle identification. See [12] for an overview and [18] for updated antihelium-3 sensitivity [19]. Exotic atom formation is unique to negatively-charged particles like antinuclei, allowing for rejection of abundant nucleus events based on the absence 

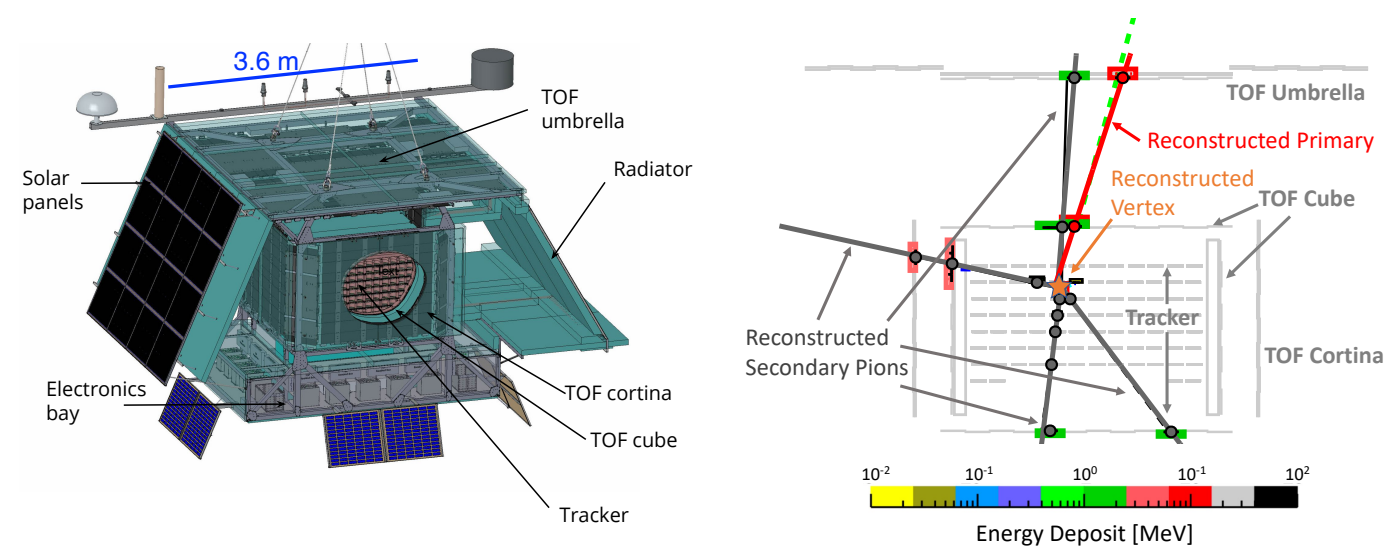

Figure 1: Left: a mechanical drawing of the GAPS instrument shows the outer TOF umbrella and cortina and the inner TOF cube. The cut-away panels reveal the layers of detectors in the $\mathrm{Si}(\mathrm{Li})$ tracker and X-ray spectrometer. The electronics bay is located beneath the sensitive material while the solar panels and radiator for the oscillating heat pipe thermal system are to the side, minimizing the mass directly above the science payload. Right: a reconstructed signal event (simulated with $\beta=0.37$ ) shows the primary antiproton track (simulated green, reconstructed red), 4 secondary pion tracks (simulated black, reconstructed dark gray), and the annihilation vertex (orange star). The colored boxes represent energy depositions in sensitive detector volumes, the largest of which (red) are on the primary track. The detector materials are in light gray.

of the distinct exotic atom signature. Further, exotic atom-based particle identification does not require a magnet, enabling a large-acceptance payload within the mass constraints of balloon flight.

Figure 1 illustrates the GAPS instrument, which consists of a $1.6 \mathrm{~m} \times 1.6 \mathrm{~m} \times 1 \mathrm{~m}$ tracker and X-ray spectrometer instrumented with $\sim 10002.5 \mathrm{~mm}$-thick lithium-drifted silicon ( $\mathrm{Si}(\mathrm{Li})$ ) detectors in 10 layers, surrounded by a 2-layer plastic scintillator time-of-flight (TOF) system. Details of the instrument design and performance are in [20] and references therein.

When a low-energy particle traverses the GAPS instrument, it crosses the outer (umbrella or cortina) and inner (cube) TOF layers, which measure the kinematic variable $\beta=v / c$, where $v$ is the velocity and $c$ is the speed of light. It then slows down via $d \mathrm{E} / d \mathrm{x}$ losses in the $\mathrm{Si}(\mathrm{Li})$ layers. Once the KE of a negatively-charged antinucleus is similar to the atomic binding energies, it is captured by a nucleus (typically $\mathrm{Si}$ or $\mathrm{Al}$ ) within the tracker volume, forming an exotic atom in an excited state. The exotic atom de-excites, producing X-rays, and then annihilates with the host nucleus, producing secondary hadrons which form tracks in the $\mathrm{Si}(\mathrm{Li})$ detectors and TOF paddles.

Positively charged nuclei do not form exotic atoms and are mostly rejected by the trigger [21] due to their low number of tracks through the TOF. However, with increasing $\beta$, the cross section for hard interactions with target nuclei increases relative to that of $d \mathrm{E} / d \mathrm{x}$ energy loss. For $\beta \gtrsim 0.4$, hard interactions are an increasingly important background. Because nuclei outnumber antiprotons by $>10^{6}$ to 1 in this energy range, a powerful identification procedure has been implemented based on kinematic differences between the hard nucleus-nucleus interactions and exotic atom annihilations.

\section{The GAPS Cosmic Antiproton Spectrum}

The figure of merit for sensitivity to cosmic particles is the acceptance $\Gamma_{a}\left[\mathrm{~m}^{2} \mathrm{sr}\right]$, which describes the physical extent of the instrument folded with energy- and angle-dependent efficiency 
of the selection criteria, for particles of species $a$. Given selection criteria, the expected number $N_{a}(E, \Delta E, \theta)$ of particles of species $a$ in a KE bin of width $\Delta E$ centered on $E$ is calculated as:

$$
N_{a}(E, \Delta E, \theta)=\Phi_{a}(E, \theta) \cdot \Gamma_{a}(E, \theta) \cdot T \cdot \Delta E .
$$

Eq. (1) shows that $N_{a}(E, \Delta E, \theta)$ over the energy range $\Delta E[\mathrm{GeV}]$ at zenith angle $\theta$ depends on the predicted flux $\Phi_{a}(E, \theta)\left[\mathrm{s}^{-1} \mathrm{~m}^{-2} \mathrm{sr}^{-1}(\mathrm{GeV} / n)^{-1}\right]$ and measurement time $T[\mathrm{~s}]$ as well as $\Gamma_{a}(E, \theta)$.

\subsection{The GAPS Detector Simulation and Event Reconstruction}

This study uses a Geant4-based [22] simulation of the GAPS instrument. To evaluate the angle and energy dependence of the instrument and analysis acceptance, particles of all signal and background species were simulated isotropically with uniform velocity from $0.1<\beta<1$, and their energy depositions in the sensitive materials were recorded and folded with the timing and energy resolution of each detector. Trigger conditions [21] requiring at minimum 8 energy depositions in the TOF material (implying a minimum number of tracks) and at least two large energy depositions consistent with $d \mathrm{E} / d \mathrm{x}$ from a low-energy particle, were applied to each simulated event.

Figure 1 shows a reconstructed signal event generated with $\beta=0.37$. For all events passing the trigger conditions, the reconstruction associates recorded energy depositions with the primary particle track, the annihilation vertex, and the secondary tracks [23]. See also [26] for the current reconstruction technique and [27] for development of machine learning-based reconstruction.

\subsection{The Antiproton Event Identification}

The antiproton analysis was performed as a $\beta$-dependent likelihood analysis on simulated events using variables obtained in event reconstruction. All reconstructed events were first required to meet a set of pre-selection criteria. Events were rejected if the reconstruction failed to converge, if the vertex was reconstructed outside of the tracker volume, if the particle path between the TOF paddles was $<90 \mathrm{~cm}$. The pre-selection also required the energy deposition pattern on the primary track to be consistent with a single slow particle (to reject events where a secondary particle was reconstructed in the primary particle track), with maximum energy deposition consistent with $Z=1$.

Figure 2 shows distributions for events passing the pre-selection criteria, for four of the event variables used in this analysis. For background studies, we focus on proton and $\alpha$ events, as these are the most abundant backgrounds in terms of their cosmic fluxes. Heavier nuclei are rejected by the energy deposition cuts in the pre-selection, while deuterium and helium-3 nuclei are less abundant and effectively alongside protons and $\alpha$ particles, respectively.

The total number of hits in the tracker and TOF is associated with the multiplicity of secondary tracks. It is particularly effective at low $\beta \lesssim 0.4$, where hard interactions (the dominant background event type) are lower in energy producing fewer secondary tracks, and where exotic atom annihilation is the most common signal event type, though even at higher energies, antiproton events still produce larger particle multiplicity than background events. Meanwhile, the average energy deposition over all detector hits is larger for higher-charge particles including $\alpha \mathrm{s}$ due to the larger $d \mathrm{E} / d \mathrm{x}$ on the primary track. The distribution is also broader for proton-nucleus interactions relative to antiprotonnucleus annihilations, as fewer and slower secondaries arise from proton-the former.

The other variables in Figure 2 pertain to the distribution of secondary tracks. The mean $\beta$ of the secondary tracks depends on the production of secondary pions relative to secondary protons, 

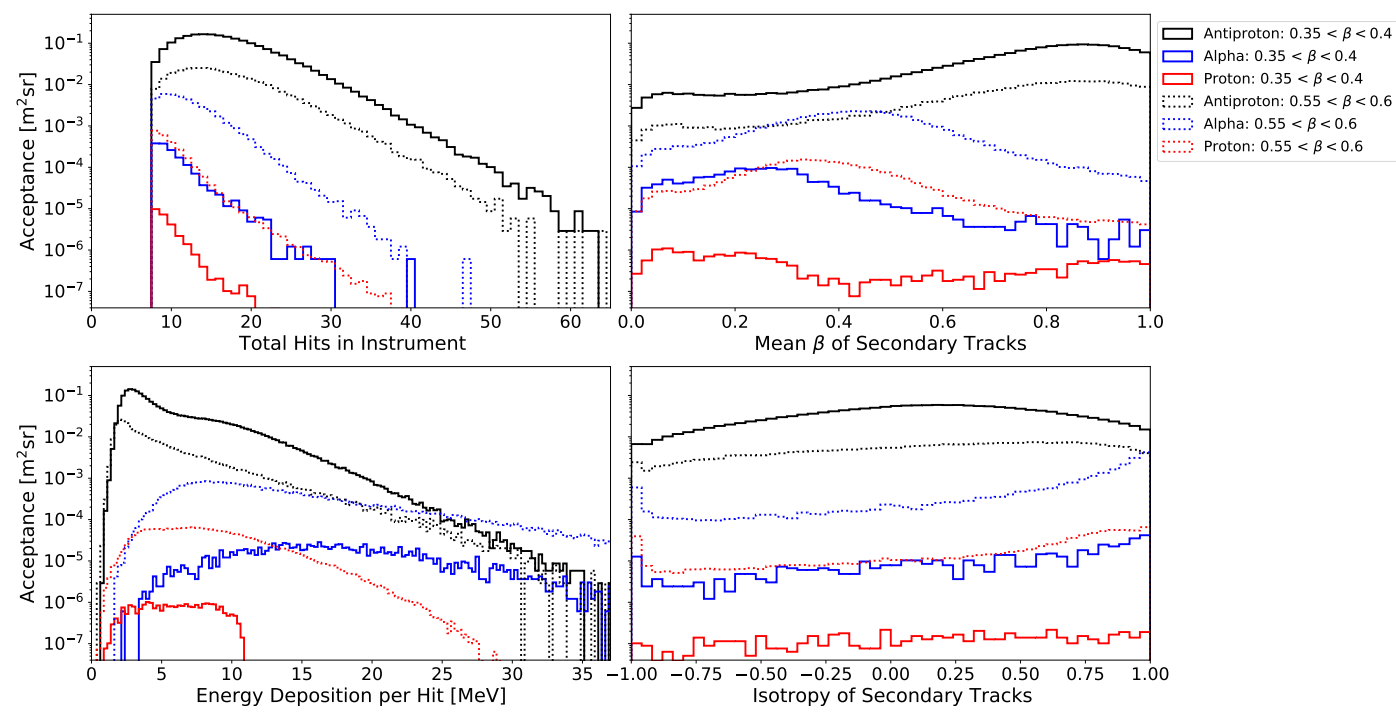

Figure 2: Distributions of four event variables, the total hits in the instrument (top left), the mean $\beta$ of the secondary tracks (top right), the mean energy deposition per hit (lower left), and the isotropy of the secondary tracks (lower right), are shown for antiproton signal (black) and proton (red) and alpha (blue) background events passing the pre-selection criteria. Distributions are shown for two $\beta$ regimes, $0.35<\beta<0.40$ (solid lines) and $0.55<\beta<0.60$ (dotted lines), to illustrated the changing morphology due to increasing importance of in-flight annihilation at higher $\beta$.
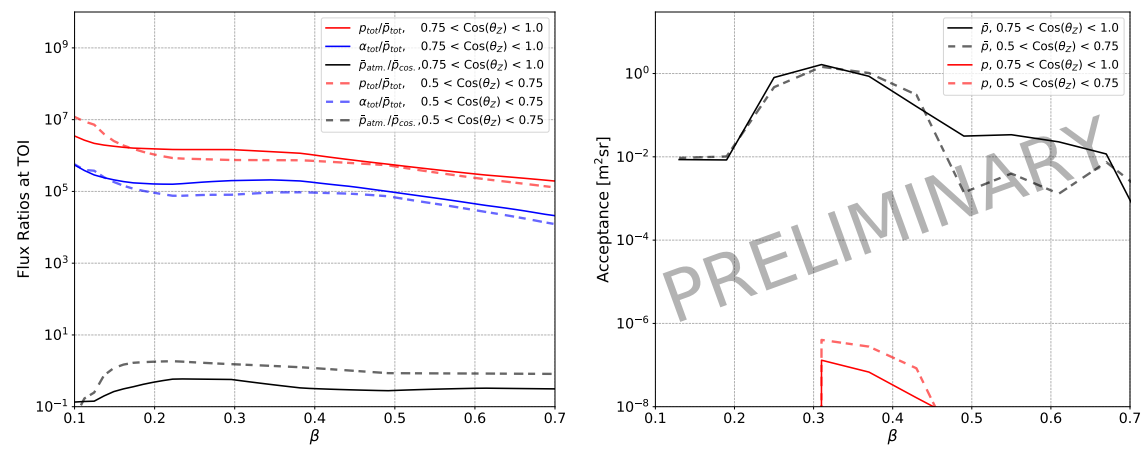

Figure 3: Left: Expected flux for the most abundant nucleus species relative to the total antiprotons, as well as atmospheric relative to cosmic antiprotons, are shown in two zenith angle ranges for TOI, $37 \mathrm{~km}$ above Antarctica. Right: Acceptance after all analysis criteria is shown for antiprotons (black) and protons (red) for two $\beta$ bins, both of which achieve the required $10^{6}$ acceptance ratio for antiprotons relative to protons.

which are produced at lower velocity. This variable is thus smaller in nucleus-nucleus interactions, which, unlike antinuclei, must produce secondary baryons to conserve baryon number. Meanwhile, the isotropy of secondary tracker hits variable measures the average cosine of the angle between each secondary hit in the tracker and the primary momentum direction. In-flight annihilation events are boosted in the primary momentum direction and thus have more extreme isotropies.

The likelihood was constructed using seven event variables including those in Figure 2. The probability distribution $P_{i}^{a}(\beta)$ for each event variable $i$ for each species $a$ was derived from simula- 
tions. Then, $P_{i}^{a}(\beta)$ was evaluated at the reconstructed $\beta_{\text {rec }}$ to obtain the probability that a particular event drawn from the distribution of variable $i$ for species $a$. The likelihood $L$ for each event was calculated as in Eq. (2), where $\mathcal{P}^{a}$, the probability that an event is drawn from the distributions of particle species $a$, is the geometric mean over all event variables $i$, of $P_{i}^{a}\left(\beta_{\text {rec }}\right)$ as shown in Eq. (2):

$$
L=\frac{\mathcal{P}^{\bar{p}}}{\mathcal{P}^{\bar{p}}+\mathcal{P}^{p}+\mathcal{P}^{\alpha}}, \quad \mathcal{P}^{a}=\sqrt[N]{\prod_{i}^{N} P_{i}^{a}\left(\beta_{\mathrm{rec}}\right)}
$$

The analysis cuts were based on the natural $\operatorname{logarithm},-\ln (L)$ of the ratio, where a low $-\ln (L)$ indicates a high probability of being an antiproton event.

Figure 3 shows the acceptance $\Gamma(E, \theta)$ for antiprotons and protons resulting from the likelihood analysis using these variables. The proton acceptance is $<10^{-6}$ of the antiproton acceptance over the range of $0.25<\beta<0.6$. The requirement on the $\alpha$ rejection is a factor of $\sim 10$ lower than for protons due to the different flux levels. Initial tests indicate that this level of $\alpha$ rejection is achievable over the same $\beta$ range. We expect that the $\alpha$ background will be lower than the proton background, with final results in an upcoming publication.

\subsection{Particle Fluxes at Float Altitude}

The fluxes at the top of the instrument (TOI, $37 \mathrm{~km}$ altitude) were simulated for signal and background species using the Geant4-based atmospheric simulation tool PLANETOCOSMICS [24]. TOA fluxes were based on conventional GALPROP [25] diffusion modulated by solar potential $\phi=$ $500 \mathrm{MV}$, expected for flight in December 2022. Geomagnetic modulation was $\sim 60-80 \%$. Cosmic particles traversing the atmosphere suffer energy- and zenith-dependent attenuation, as some of the flux is absorbed, such that the fluxes at TOI are related to TOA by a survival probability $S(E, \theta)$. Particles that survive to TOI experience energy- and zenith-dependent energy loss, $L\left(E_{\mathrm{TOI}}, \theta_{\mathrm{TOI}}\right)$, from ionization and excitation. The resulting TOI fluxes are non-isotropic due to the increased atmospheric column density at wide angles, and include both fluxes of cosmic origin and particles produced in interactions of cosmic particles with the atmosphere, illustrated by the ratios in Figure 3.

\subsection{Toward Antiproton Spectra at Float Altitude and the Top of the Atmosphere}

The TOA spectrum is estimated by scaling according the the survival probability $S\left(E_{\mathrm{TOA}}, \theta_{\mathrm{TOI}}\right)$ and energy loss $L\left(E_{\mathrm{TOI}}, \theta_{\mathrm{TOI}}\right)$ from Section 3.3:

$$
\Phi_{\mathrm{TOA}}\left(E_{\mathrm{TOA}}\right)=\Phi_{\mathrm{TOI}}\left(E_{\mathrm{TOI}}, \theta_{\mathrm{TOI}}\right) \cdot\left(\Delta E_{\mathrm{TOI}} / \Delta E_{\mathrm{TOA}}\right) / S\left(E_{\mathrm{TOA}}, \theta_{\mathrm{TOI}}\right),
$$

for $E_{\mathrm{TOA}}=E_{\mathrm{TOI}}+L\left(E_{\mathrm{TOI}}, \theta_{\mathrm{TOI}}\right)$. From the first 35-day flight, we expect a TOA spectrum with about 7 bins in the range of $0.1<\mathrm{KE}<0.25$, each with $\sim 30 \%$ statistical uncertainty. With data from the full GAPS observation (three LDB flights), the statistical uncertainty will decrease to $\sim 10-20 \%$. More details will be shown in a forthcoming publication.

\subsection{Sources of Systematic Uncertainty}

While the analysis effectively rejects protons and heavier nuclei such that subtraction of these fluxes is not anticipated to yield a significant systematic error, antiprotons produced in the atmosphere are identical to cosmic antiprotons in the detector and represent an irreducible background. 
Errors in the atmospheric modeling impact the systematic error of the cosmic antiproton result. Fortunately, at float altitude, atmospheric antiprotons dominate the total antiproton flux at shallow zenith angles, while cosmic antiprotons dominate at steep zenith angles. Using the wide angular acceptance of the GAPS instrument, the dependence of the antiproton flux on the zenith angle can be used to study the atmospheric antiproton component, reducing systematics associated with this intrinsic background. Translating the TOI flux to TOA introduces additional systematic uncertainty associated with the atmospheric simulations. Any error in the survival probability $S(E)$ from TOA to TOI or the typical energy loss $\Delta \beta_{T O A \rightarrow T O I}(\beta, \theta)$ between TOA and TOI would lead to systematic errors in the TOA measurement. More detailed atmospheric studies are ongoing.

An example of instrumental systematic error arises from the $\beta$ resolution. With $\sigma_{\beta} / \beta \sim 5-10 \%$ (RMS) and bins of width $\Delta \beta \sim 0.05$, a systematic error arises due to bin-to-bin migration.

\section{Conclusion}

In this contribution, we have demonstrated the GAPS Experiment sensitivity to antiprotons. In one 35-day Antarctic flight, GAPS will measure a high-statistics cosmic antiproton spectrum in the $\mathrm{KE}$ range of $0.03-0.23 \mathrm{GeV} / n$ at float altitude, corresponding to $\sim 0.1-0.26 \mathrm{GeV} / n$ at TOA. This measurement will inform indirect DM searches and is sensitive to evaporating local primordial black holes and Galactic propagation models. Moreover, using data from the first flight, the antiproton analysis will validate the exotic atom particle identification method for all antinuclei, facilitating confidence in the GAPS rare event searches for antideuterons and antihelium-3.

\section{Acknowledgments}

This work is supported in the U.S. by NASA APRA grants (NNX17AB44G, NNX17AB45G, NNX17AB46G, and NNX17AB47G), in Japan by JAXA/ISAS Small Science Program FY2017, and in Italy by Istituto Nazionale di Fisica Nucleare (INFN) and the Italian Space Agency through ASI INFN agreement No. 2018-28-HH.0: "Partecipazione italiana al GAPS - General AntiParticle Spectrometer". F. Rogers is supported by the National Science Foundation (NSF) Graduate Research Fellowship under Grant No. 1122374. P. von Doetinchem received support from the NSF under award PHY-1551980. H. Fuke is supported by JSPS KAKENHI grants (JP17H01136 and JP19H05198) and Mitsubishi Foundation Research Grant 2019-10038. K. Perez and M. Xiao are supported by Heising-Simons award 2018-0766. Y. Shimizu receives support from JSPS KAKENHI grant JP20K04002 and Sumitomo Foundation Grant No. 180322. Technical support and advanced computing resources from the University of Hawaii Information Technology Services Cyberinfrastructure are gratefully acknowledged. This research was done using resources provided by the Open Science Grid [29, 30], which is supported by the NSF award No. 2030508.

\section{References}

[1] P. von Doetinchem, K. Perez, et al., JCAP08(2020)035 [2002.04163].

[2] M. Aguilar et al., Phys. Rev. Lett. 117 (9) (2016) 091103. 
[3] BESS Collaboration, Adv. in Space Res. 512 (2013) 227-233

[4] K. Abe et al., Phys. Rev. Lett. 1085 (2012) 051102 [1107.6000].

[5] O. Adriani et al., JETP Letters 96 (10) (2013) 621-627.

[6] M. Y. Cui et al., Phys. Rev. Lett. 118(2017) 191101 [1610.03840].

[7] A. Cuoco et al., Phys. Rev. Lett. 118(2017) 191102 [1610.03071].

[8] A. Reinert and M. W. Winkler, JCAP 1801(2018) 055 [1712.00002].

[9] A. Cuoco et al., Phys. Rev. D 99(2019) 103014 [1903.01472].

[10] I. Cholis et al., Phys. Rev. D 99(2019) 103026 [1903.02549].

[11] M. Boudaud et al., Phys. Rev. Research 2 (2020) 023022 [1906.07119].

[12] M. Xiao et al., This conference Proceedings of Science, 2021.

[13] K. Mori et al., Ap. J. 566 (2002) 604 [astro-ph/0109463].

[14] C. J. Hailey, New J. Phys. 11 (2009) 105022.

[15] GAPS collaboration, Adv. Space Res. 51 (2013) 290.

[16] T. K. Gaisser and E. H. Levy, Phys. Rev. D 10 (1974) 1731.

[17] T. Aramaki et al., Astropart. Phys. 59 (2014) 12-17 [1401.8245].

[18] A. Stoessl et al., This conference Proceedings of Science, 2021.

[19] N. Saffold et al., Astropart. Phys. 130 (2021) 102580 [2012.05834].

[20] S. Quinn et al., This conference Proceedings of Science, 2021.

[21] S. Quinn et al., in Proceedings of Science ICRC 2019 [1912.01675].

[22] S. Agostinelli et al., NIM A 5063 (2003) 250-303.

[23] R. Munini et al., submitted to Astropart. Phys. (2021).

[24] L. Desorgher et al., 36th COSPAR Scientific Assembly, 36, 2361 (2006).

[25] A. W. Strong et al., 1092 Ann. Rev. Nuc. Part. Sci. 57 (2007) 285-327.

[26] A. Tiberio et al., This conference Proceedings of Science, 2021.

[27] N. Marcelli et al., This conference Proceedings of Science, 2021.

[28] A. Barrau et al., A\&A 388 (2002) 676 [astro-ph/0112486].

[29] R. Pordes et al., in J. Phys. Conf. Ser. 78, (2007) 012057.

[30] I. Sfiligoi et al., in 2009 WRI World Congress on Computer Science and Information Engineering 2 (2009) 428-432. 


\section{Full Authors List: GAPS Collaboration}

T. Aramaki ${ }^{1}$, R. Bird ${ }^{2}$, M. Boezio ${ }^{3,4}$, S. E. Boggs ${ }^{5}$, V. Bonvicini ${ }^{3}$, D. Campana ${ }^{6}$, W. W. Craig ${ }^{7}$, E. Everson ${ }^{2}$, L. Fabris ${ }^{8}$, H. Fuke ${ }^{9}$, F. Gahbauer ${ }^{10}$, I. Garcia ${ }^{2}$, C. Gerrity ${ }^{11}$, C. J. Hailey ${ }^{10}$, T. Hayashi ${ }^{2}$, C. Kato ${ }^{12}$, A. Kawachi ${ }^{13}$, S. Kobayashi ${ }^{13}$, M. Kozai ${ }^{9}$, A. Lenni ${ }^{3,14}$, A. Lowell ${ }^{7}$, M. Manghisoni ${ }^{15,16}$, N. Marcelli ${ }^{17,18}$, B. Mochizuki ${ }^{7}$ S. A. I. Mognet ${ }^{19}$, K. Munakata ${ }^{12}$, R. Munini ${ }^{3,4}$, Y. Nakagami $^{20}$, J. Olson ${ }^{21}$, R. A. $\mathrm{Ong}^{2}$, G. Osteria ${ }^{6}$, K. Perez ${ }^{22}$, S. Quinn' ${ }^{2}$, V. Re ${ }^{15,16}$, E. Riceputi ${ }^{15,16}$, B. Roach ${ }^{22}$, F. Rogers ${ }^{22}$, J. A. Ryan ${ }^{2}$, N. Saffold ${ }^{10}$, V. Scotti ${ }^{6,23}$, Y. Shimizu ${ }^{24}$, M. Sonzogni ${ }^{15,16}$, R. Sparvoli ${ }^{17,18}$, A. Stoess $1^{11}$, A. Tiberio ${ }^{25}$, E. Vannuccini ${ }^{25}$, P. von

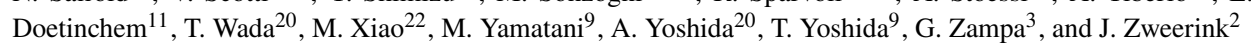

${ }^{1}$ Northeastern University, 360 Huntington Avenue, Boston, MA 02115, USA. ${ }^{2}$ University of California, Los Angeles, Los Angeles, CA 90095, USA. ${ }^{3}$ INFN, Sezione di Trieste, I-34149 Trieste, Italy. ${ }^{4}$ IFPU, I-34014 Trieste, Italy. ${ }^{5}$ University of California, San Diego, La Jolla, CA 90037, USA. ${ }^{6}$ INFN, Sezione di Napoli, I-80126 Naples, Italy. ${ }^{7}$ Space Sciences Laboratory, University of California, Berkeley, 7 Gauss Way, Berkeley, CA 94720, USA. ${ }^{8}$ Oak Ridge National Laboratory, Oak Ridge, TN 37831, USA. ${ }^{9}$ Institute of Space and Astronautical Science, Japan Aerospace Exploration Agency (ISAS/JAXA), Sagamihara, Kanagawa $252-5210$, Japan. ${ }^{10}$ Columbia University, New York, NY 10027, USA. ${ }^{11}$ University of Hawaii at Manoa, Honolulu, HI 96822 USA. ${ }^{12}$ Shinshu University, Matsumoto, Nagano 390-8621, Japan. ${ }^{13}$ Tokai University, Hiratsuka, Kanagawa 259-1292, Japan. ${ }^{14}$ Universitá di Trieste, I-34127 Trieste, Italy. ${ }^{15}$ INFN, Sezione di Pavia, I-27100 Pavia, Italy. ${ }^{16}$ Universitá di Bergamo, I-24044 Dalmine (BG), Italy. ${ }^{17}$ INFN, Sezione di Rome "Tor Vergata", I-00133 Rome, Italy. ${ }^{18}$ Universitá di Roma "Tor Vergata", I-00133 Rome, Italy. ${ }^{19}$ Pennsylvania State University, University Park, PA 16802 USA. ${ }^{20}$ Aoyama Gakuin University, Sagamihara, Kanagawa 252-5258, Japan. ${ }^{21}$ Heliospace Corporation, Berkeley, CA 94710, USA. ${ }^{22}$ Massachusetts Institute of Technology, Cambridge, MA 02139, USA. ${ }^{23}$ Universitá di Napoli "Federico II", I-80138 Naples, Italy. ${ }^{24}$ Kanagawa University, Yokohama, Kanagawa 221-8686, Japan. ${ }^{25}$ INFN, Sezione di Firenze, I-50019 Sesto Fiorentino, Florence, Italy.

Web version: gaps1.astro.ucla.edu/gaps/authors/ 